\title{
Mechanosensing regulates liver growth
}

The mechanical effects of blood flow drive hepatic growth and regeneration, according to new research published in Nature.

Over the past decade, increasing attention has been paid to the role of angiocrine signals, derived from endothelial cells, in regulating the growth and repair of many organs. This aspect is particularly true for the liver, an organ with an extensive blood supply that can regenerate more than two-thirds of its volume.

To explore how blood perfusion regulates liver growth, Eckhard Lammert and colleagues studied liver development in mice. "We used ex vivo cultivation of mouse embryos in which we either stopped the heart beat and thus the blood flow or increased it using epinephrine and atropine," explains Lammert. These experiments indicated that blood perfusion promotes liver growth. Notably, increased blood flow was associated with increased activation of the mechanosensory molecules $\beta 1$ integrin and VEGFR3. Levels of hepatocyte growth factor (HGF), a key regulator of liver growth, were also upregulated by increased blood flow.

Liver growth was markedly impaired and HGF levels were decreased when $\beta 1$ integrin or VEGFR3 were knocked out specifically in endothelial cells, despite no substantial change in the number of blood vessels. "We then showed that ex vivo perfusion of adult mouse liver also turns on HGF production and c-Met signalling as flow increases and sinusoids are dilated," continues Lammert. To confirm the specific role of mechanical forces in this effect, the investigators stretched human liver sinusoidal endothelial cells in culture; these cells released HGF and the pro-regenerative factors MMP9, TNF and IL-6.

In addition to shedding light on the fundamental mechanisms regulating liver growth and

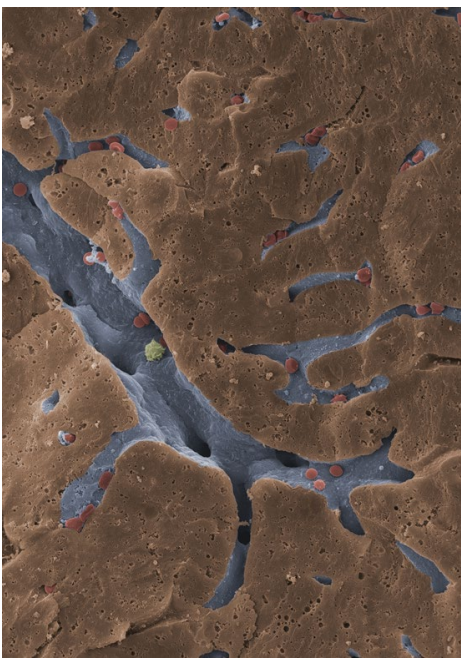

Electron micrograph showing hepatic blood vessels. Image courtesy of D. Eberhard, Heinrich Heine-University Düsseldorf, Germany and S. Köhler, Heinrich-Heine-University Düsseldorf, Germany.

...increased

blood flow was

associated

with increased

activation of

the mechano-

sensory

molecules $\beta 1$

integrin and

VEGFR3

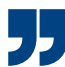

regeneration, Lammert points out that these findings could improve hepatocyte culture in vitro. A better appreciation of the pathways involved in liver regeneration might also aid understanding of diseases in which this process is impaired, such as cirrhosis.

Hugh Thomas

ORIGINAL ARTICLE Lorenz, L. et al.

Mechanosensing by $\beta 1$ integrin induces angiocrine signals for liver growth and survival. Nature 562, 128-132 (2018)

\section{New pathways in development of liver cancer}

Obesity is a risk factor for a range

of cancers, with the association

being particularly strong for

hepatocellular carcinoma (HCC).

Although it was thought that nonalcoholic steatohepatitis (NASH) and fibrosis or cirrhosis were required for the development of HCC, new research suggests that obesity can cause HCC independently of NASH and fibrosis.

Signal transducer and activator of transcription (STAT) signalling pathways are known to be involved in the development of HCC. In mice, the researchers demonstrated that hepatic STAT1 and STAT3 signalling were increased in conditions of obesity. Furthermore, STAT1 signalling was elevated in the livers of mice fed a nonalcoholic fatty liver (NAFL)-promoting diet high in fat, and was further elevated in the livers of mice fed a NASH-promoting diet. These findings were supported by human studies; STAT1 signalling was increased in patients with obesity and nonalcoholic fatty liver disease (NAFLD).

In addition, the STAT1 phosphatase TCPTP was found to be oxidized in the livers of mice fed a NAFL-promoting diet (and the oxidation was further increased in mice fed a NASHpromoting diet) and in the livers of patients with obesity and NAFLD. In mice that were deficient in TCPTP, STAT1 signalling was increased in liver cells, which led to accumulation of T cells, inflammation, NASH and fibrosis when the mice were fed a high-fat diet. By contrast, wild-type mice developed steatosis, but not fibrosis and NASH,
when fed a high-fat diet. steatosis, but not fibrosis
when fed a high-fat diet.

When STAT1 signalling was reduced in TCPTP-deficient mice fed a high-fat diet, $T$ cell recruitment was prevented and NASH and fibrosis did not develop; however, the mice did develop HCC. The authors suggest that obesity and STAT3 signalling are important for the development of HCC, as chow-fed mice did not develop HCC and reducing STAT3 signalling prevented the development of HCC.

The authors conclude that increased STAT3 signalling in conditions of obesity and NAFLD is sufficient to drive the development of HCC, even in the absence of fibrosis or cirrhosis. They suggest that it is the oxidative and inflammatory environment of the liver in NAFLD that results in HCC, rather than the development of NASH or fibrosis.

Claire Greenhill, Nature Reviews Endocrinology This article is modified from the original in to drive the development of HCC Nat. Rev. Endocrinol. Nat. Rev. Endocrinol.
(https://doi.org/10.1038/s41574-018-0129-7).

ORIGINAL ARTICLE Grohmann, M. et al. Obesity drives STAT-1-dependent NASH and STAT-3-dependent HCC. Cell https://doi.org/ 10.1016/j.cell.2018.09.053 (2018) 